\title{
Antibody response after influenza immunization with various vaccine doses: a double-blind, placebo- controlled, multi-centre, dose-response study in elderly nursing-home residents and young volunteers
}

\author{
A.M. Palache*, W.E.P. Beyer*, M.J.W.Sprenger*, N. Masurel*§, S. de Jonge ${ }^{\dagger}$, \\ A. Vardy $^{\dagger}$, B. Charpentier ${ }^{\dagger}$, J. Noury ${ }^{\infty}$, W.C.A. van Beek ${ }^{\|}$, R.J.A. Borst ${ }^{\dagger}$, \\ G.J. Ligthart", G. Keren " and E. Rubinstein
}

The dose effect $(0,10,20$ and $60 \mu \mathrm{g})$ of influenza subunit vaccine on the antibody response was investigated in nursing-home residents and young controls. The vaccine antigens were: A/Taiwan/1/86(HINI), A/Sichuan/2/87(H3N2) and B/Beijing/1/87. For the influenza $B$ antigen, the post-GMT and the 'percentage protective titre' increased significantly both in the young controls and nursing-home residents. No dose effect was observed for the A/Taiwan, and a minor dose effect for A/Sichuan. All vaccine doses were well tolerated by both groups. We conclude from our data that higher vaccine doses may result in a better antibody response against some antigens but not against others. Therefore, in general, increasing the vaccine dose is no adequate method to improve the antibody response.

Keywords: Influenza: immunization; antibody; dose; elderly; nursing-home residents

\section{INTRODUCTION}

Worldwide, annual immunization is recommended for individuals, including nursing-home residents, at high risk of serious complications or death from influenza infections ${ }^{1,2}$. Although various authors have convincingly shown that influenza vaccinations modify influenza by the reduction of influenza- and pneumonia-associated hospitalizations ${ }^{3-6}$, vaccination studies with inactivated influenza vaccines have shown disappointing protection against infections in nursing-home residents ${ }^{7-9}$. These modest results are generally considered to be due to an age-associated decline in immune response following a standard dose of vaccination ${ }^{7,10}$, although this assumption is disputed by several authors ${ }^{11,12}$.

In an attempt to increase the immune response in the elderly and hence to enhance protection against influenza

*Department of Virology and WHO Influenza Center, Erasmus University Rotterdam, PO Box 1738, 3000 DR Rotterdam, The Netherlands. ${ }^{\dagger}$ Duphar BV, Weesp, The Netherlands. ${ }^{\ddagger}$ Hôpital Bicetre, Paris, France. ${ }^{\infty}$ Innopharm, Paris, France. "Academic Hospital, Leiden, The Netherlands. 'Tel Hashomer Hospital, Tel Aviv University, Israel. ${ }^{\S}$ To whom correspondence should be addressed. (Received 13 June 1991; revised 9 December 1991; accepted 16 December 1991) infections, several authors have investigated the effect of high-dose influenza $B$ virus vaccines on the humoral response in ambulatory elderly nursing-home residents and young controls ${ }^{13-15}$.

Since these studies did not yield a consistent answer as to whether an increased dose would be beneficial or not, we performed a double-blind, placebo-controlled, randomized, multi-centre, dose-response study in elderly nursing-home residents and young controls. We investigated the antibody response following doses of 10 , 20 and $60 \mu \mathrm{g} \mathrm{HA} /$ strain trivalent subunit vaccine.

\section{MATERIALS AND METHODS}

Study population

We immunized 282 young volunteers (mean age 21; range $17-47$ years) and 262 elderly (mean age 80 ; range 68-99 years) nursing-home residents with placebo (saline) or trivalent influenza subunit vaccine of various concentrations, between October and December 1988. For both age groups, all treatment groups were comparable with regard to their age and sex distribution.

Each age group was studied in different study centres. The young volunteers were university students from Rotterdam, The Netherlands (centre 1, $n=140$ ) and 
Paris, France (centre 2, $n=142$ ). The elderly were recruited either in 20 nursing-homes spread throughout the western part of The Netherlands (centre $3, n=122$ ) or in a single nursing-home in Tel Aviv, Israel (centre 4, $n=140$ ). Fifty-eight subjects from centre 4 had been immunized against influenza at least once during the 5 years preceding the current study. All other participants had not been vaccinated against influenza during this period.

Patients taking immunosuppressive medications such as corticosteroids and cytostatics were excluded. Informed consent prior to the study was given by all participants except for nursing-home residents with dementia. In the Israeli group, relatives of subjects with dementia gave informed consent, whereas in the Dutch group, subjects with dementia were excluded from the study. The study was approved by local ethics committees.

\section{Vaccines}

Experimental trivalent influenza subunit vaccines (Duphar BV, The Netherlands) contained 10, 20 or $60 \mu \mathrm{g}$ HA for each of the following antigens: B/Beijing/1/87, A/Taiwan/1/86 (H1N1) and A/Sichuan/2/87 (H3N2). For each antigen, the different dosages were drawn from the same production lot. Each treatment group received a $0.5 \mathrm{ml}$ intramuscular injection of saline or the experimental trivalent vaccine.

The experimental vaccines were produced according to the manufacturer's standard procedures for its commercially available subunit vaccine. Briefly, virus was propagated for 2 days on chicken eggs and, after harvesting, inactivated by formaldehyde and disrupted by cetyltrimethyl ammonium bromide. Membrane proteins were isolated by zonal ultracentrifugation. The final preparation contained $0.01 \%$ sodium ethylmercurithiosalicyle, 0.00625 IU polymyxin, and $0.00625 \mu \mathrm{g}$ neomycin per dose.

\section{Study design}

Vaccinations were given on a double-blind basis according to a treatment randomization scheme. From each volunteer, a $10 \mathrm{ml}$ blood sample was drawn just prior to vaccination and again 3 weeks thereafter.

\section{Laboratory investigations}

Sera were separated after blood collection, kept frozen at $-20^{\circ} \mathrm{C}$, and transported fom each study centre to the Department of Virology, Rotterdam, The Netherlands for laboratory investigation. On arrival in Rotterdam, all sera were recoded according to a new randomization scheme in order to achieve blindness in the antibody determinations. Each day, the pre- and postimmunization sera of 12 subjects from each study centre were analysed. Influenza virus strains for titrations were propagated in embryonated 12-day-old chicken eggs. Because of the low avidity of the influenza B viruses, infectious egg fluids of this strain were treated with ether according to the method of Berlin et al. ${ }^{16}$ and the aqueous phase was used in the serological tests. Pre- and postimmunization haemagglutinin-inhibition (HI) titres were simultaneously determined in duplicate by standard methods ${ }^{17}$. Titres were expressed as the reciprocals of the dilution showing $50 \%$ haemagglutination inhibition with 3 haemagglutination units of the antigen. From the results of the determinations per serum and per antigen, the geometric means were used for further calculations. Negative titres $(<9)$ were arbitrarily regarded as 5 . With the method used, protection against infection is assumed to be associated with a $\mathrm{HI}$ titre of $\geqslant 100$ for influenza $A$ strains $^{18,19}$. For ether-treated influenza $B$ strains, no such protection threshold is known. As previously ${ }^{19}$, for this study a titre threshold of 200 was chosen.

\section{Clinical investigations}

Each participant received a standard symptom questionnaire for the evaluation of reactogenicity of the vaccines during the first $48 \mathrm{~h}$ after vaccination. Symptoms were divided into local reactions such as redness, swelling, itching or pain on the site of injection and systemic reactions such as fever, headache, sweating or malaise. In the elderly group, the same assessments were made by the nursing-home physicians rather than by the subjects themselves.

\section{Statistical analysis}

As stated above, the $\mathrm{HI}$ antibody titre per subject, time point (pre-/postimmunization) and antigen were calculated as the geometric mean of the two corresponding laboratory determinants. These titre values were used to derive four further variables for statistical analysis: the geometric mean titre (GMT), representing the geometric means of the individual titre values; the proportion of subjects with HI-antibody titres above the assumed protective level (percentage protective titre); the geometric mean fold increase (MFI) and the proportion of subjects with a fourfold or greater titre increase (percentage responders).

The titre values and MFI were subjected to a logarithmic transformation and analysed using analysis of variance and covariance (PROC GLM in SAS $)^{20}$. The percentage protective titre and percentage of responders were subjected to a logit transformation and analysed using logistic regression (PROC LOGISTIC in SAS).

For the analysis of the vaccinated groups, the transformed variables were related by an additive, linear model to the factors age group, centre within age group, dose, interaction between dose and age group, and interaction between dose and centre within age group. In addition, the analyses of postimmunization titre and percentage protective titre included pre-immunization titre as a covariate, since it is recognized that preimmunization titre has an influence on postimmunization values $^{2 i}$. Because MFI and percentage of responders 'correct' for pre-immunization titre, no covariate was included in the analyses of these variables.

The analyses performed provide for tests of statistical significance of the contribution of the factors listed above to the model describing the response variable. They also provide coefficients so that the value of the response variable can be predicted from an equation including those factors determined as significant.

The analysis of the placebo group followed the methods outlined above, but included only age group and centre (and pre-immunization titre where appropriate) in the model. In the description of results, the following convention is used. Borderline statistically significant indicates a $p$ value between 0.10 and 0.05 ; statistically significant indicates a $p$ value between 0.05 
and 0.01 ; and highly statistically significant indicates a $p$ value less than 0.01 .

Since the GMT and percentage protective titre are considered clinically the most meaningful markers for efficacy, and the analysis of the other variables showed qualitatively similar results as the GMT and percentage protective titre, only these latter parameters are discussed in this paper. Other parameters, such as protection rate, response rate and seroconversion rate, as defined by Beyer et al. ${ }^{11}$, were calculated and analysed, but again qualitatively similar results were obtained and are therefore not presented in this paper.

Since increased antibody titres would result in an extended period of effective protection against influenza infections ${ }^{22,23}$, we have also analysed the frequency distribution of subjects within discrete postimmunization titre intervals for all antigens. This analysis did not reveal additional relevant dose effects and is therefore also not presented in this paper.

\section{RESULTS}

\section{Pre- and postimmunization antibody status of} placebo-treated groups

The pre- and postimmunization GMT and percentage protective titre of the placebo-treated groups in the four study centres for the three vaccine antigens are presented in Table 1. Although some highly statistically significant study centre differences within each age category are apparent, there was virtually no antibody titre rise in each of the placebo groups, so that no placebo response corrections were needed in the analysis of the actively immunized groups.

\section{Prevaccination antibody status}

Prevaccination GMT and percentage protective titre for the four centres and the three vaccine antigens for the actively immunized subjects are presented in Table 2 . The average prevaccination GMT against the $B /$ Beijing $/ 1 / 87$ (B/B) antigen was approximately 30 for both age groups. However, statistically significant study centre differences within each age group were revealed. Within the young age group, the participants of centre 1 (students in Rotterdam ) had a highly statistically significantly higher prevaccination GMT than those of centre 2 (students in Paris). The nursing-home residents in Tel Aviv (centre 4) had a statistically significantly higher prevaccination GMT than their Dutch counterparts (centre 3). These centre differences were not reflected by the percentage protective titre values which were $10 \%$ on average.

Antibodies against the (A/Taiwan/1/86 (H1N1)) $(\mathrm{A} / \mathrm{T})$ strain were low on average (postvaccination $\mathrm{GMT}=8$, percentage protective titre $=3 \%$ ). The study centre differences for this antigen are considered irrelevant.

The proportion of subjects with protective antibody titres against the (A/Sichuan/2/87 (H3N2)) (A/S) strain was much greater than against the other two antigens. Both age and centre differences were obvious. On average, the nursing-home residents had a significantly higher preimmunization GMT (82) than the students (61). Also the pre-immunization GMT centre differences within study populations were highly statistically significant (GMT young: 70 , centre $1 ; 52$, centre 2 ; GMT nursing-home residents: 65 , centre 3; 99 , centre 4 ).

The generally higher antibody level of centre 4 could not be explained by the preimmunization titres of the 58 previously vaccinated subjects in this study centre. When

Table 1 Pre- and postimmunization antibody status of placebo-treated subjects

\begin{tabular}{|c|c|c|c|c|c|c|c|c|}
\hline \multirow[b]{2}{*}{ Group } & & \multirow[b]{2}{*}{ Number } & \multicolumn{3}{|c|}{$\begin{array}{c}\text { GMT } \\
\text { (pre/post vaccination) }\end{array}$} & \multicolumn{3}{|c|}{$\begin{array}{l}\text { Percentage-protective titre } \\
\text { (pre/post vaccination) }\end{array}$} \\
\hline & & & $\mathrm{B} / \mathrm{B}^{\mathrm{a}}$ & $\mathrm{A} / \mathrm{T}^{\mathrm{b}}$ & $A / S^{c}$ & $B / B$ & $\mathrm{~A} / \mathrm{T}$ & $A / S$ \\
\hline \multirow[t]{3}{*}{ Young: } & Centre 1 & 34 & $33 / 29$ & $9 / 9$ & 8391 & $9 / 6$ & $3 / 3$ & $41 / 44$ \\
\hline & Centre 2 & 36 & $23 / 25$ & $6 / 7$ & $47 / 48$ & $6 / 8$ & $3 / 3$ & $25 / 31$ \\
\hline & Total & 70 & $27 / 27$ & $8 / 8$ & $62 / 66$ & $7 / 7$ & $3 / 3$ & $33 / 37$ \\
\hline \multirow[t]{3}{*}{ Elderly: } & Centre 1 & 30 & $22 / 22$ & $5 / 6$ & $71 / 76$ & $7 / 13$ & $0 / 0$ & $43 / 40$ \\
\hline & Centre 2 & 35 & $85 / 82$ & $11 / 11$ & $95 / 87$ & $23 / 29$ & $6 / 3$ & $57 / 63$ \\
\hline & Total & 65 & $45 / 45$ & $8 / 8$ & $100 / 100$ & $15 / 22$ & $3 / 2$ & $51 / 52$ \\
\hline
\end{tabular}

${ }^{a} \mathrm{~B} / \mathrm{B}, \mathrm{B} /$ Beijing $/ 1 / 87$

${ }^{b} \mathrm{~A} / \mathrm{T}, \mathrm{A} /$ Taiwan $/ 1 / 86(\mathrm{H} 1 \mathrm{~N} 1)$

${ }^{c} \mathrm{~A} / \mathrm{S}, \mathrm{A} / \mathrm{Sichuan} / 2 / 87$ (H3N2)

Table 2 Prevaccination antibody status, according to age and study centre

\begin{tabular}{|c|c|c|c|c|c|c|c|c|}
\hline \multirow[b]{2}{*}{ Group } & & \multirow[b]{2}{*}{ Number } & \multicolumn{3}{|c|}{ Prevaccination $\mathrm{GMT}^{\mathrm{B}}$} & \multicolumn{3}{|c|}{$\begin{array}{c}\text { Prevaccination } \\
\text { percentage-protective titre }\end{array}$} \\
\hline & & & $\mathrm{B} / \mathrm{B}$ & $\mathbf{A} / \mathrm{T}$ & $A / S$ & $\mathbf{B} / \mathbf{B}$ & $\mathbf{A} / \mathrm{T}$ & $\mathrm{A} / \mathrm{S}$ \\
\hline \multirow[t]{3}{*}{ Young: } & Centre 1 & 105 & 41 & 9 & 70 & 8 & 3 & 35 \\
\hline & Centre 2 & 107 & 22 & 8 & 52 & 10 & 4 & 32 \\
\hline & Total & 212 & 30 & 8 & 61 & 9 & 3 & 33 \\
\hline \multirow[t]{3}{*}{ Eldery: } & Centre 3 & 92 & 24 & 6 & 65 & 10 & 0 & 37 \\
\hline & Centre 4 & 105 & 38 & 9 & 99 & 12 & 7 & 49 \\
\hline & Total & 197 & 31 & 8 & 82 & 11 & 4 & 43 \\
\hline \multicolumn{2}{|c|}{ All participants } & 409 & 30 & 8 & 70 & 10 & 3 & 38 \\
\hline
\end{tabular}

asee footnote to Table 1 
comparing the dose groups with regard to their prevaccination antibody status, no relevant differences could be seen (see Table 3, columns 'pre GMT' and 'pre $\%$ protective titre').

\section{Antibody response after active immunization in both age groups}

In Table 3 are presented the $\mathrm{HI}$ antibody response expressed by postvaccination GMT and percentage protective titre, after active immunization with 10,20 or $60 \mu \mathrm{g}$ HA per vaccine antigen, for both age groups. For both parameters, actual and predicted values are given. Predicted values were calculated from actual values by adjusting actual values for prevaccination titres by an ANCOVA procedure (postvaccination GMT) or logistic regression (percentage protective titre).

For the B/B antigen, a highly significant dose effect on the postvaccination GMT could be demonstrated for both age groups. After correction for prevaccination titres, there was a GMT increase of 1.4 fold when increasing the dose from $10 \mu \mathrm{g}$ to $20 \mu \mathrm{g}$, and of 2.1 fold when increasing the dose from $10 \mu \mathrm{g}$ to $60 \mu \mathrm{g}$, in both age groups. The positive dose-response relation was also expressed by the corrected percentage protective titre parameter which rose $9 \%$ (young) or $15 \%$ (nursinghome residents) when increasing the dose from $10 \mu \mathrm{g}$ to $20 \mu \mathrm{g}$. The additional gain when increasing the dose from $20 \mu \mathrm{g}$ to $60 \mu \mathrm{g} \mathrm{(4 \%} \mathrm{and} 8 \%$, respectively) was small. In all dose groups, the young subjects showed a better response than the nursing-home residents. However, this highly statistically significant difference in response between the two populations was due to the higher values in all dose groups of centre 1 (Rotterdam) compared with centre 2 (Paris; GMT 1.8 fold; percentage protective titre $14-28 \%$ ). The response in centre 2 (young) and centres 3 and 4 (nursing-home residents) did not differ significantly.

In contrast to the influenza $B$ strain, the $A / T$ antigen induced a poor antibody response, particularly in the nursing-home residents, and did not show a dose effect in either age group. Postvaccination GMT values greater by $1.3-1.5$ fold and $9 \%$ greater percentage protective titre values were observed in the $60 \mu \mathrm{g}$ groups compared with the $10 \mu \mathrm{g}$ groups. For the predicted values the dose effect was even less.

The young age group responded significantly better in all dose groups than the nursing-home residents: 2.9 -fold greater postvaccination GMT values and $28 \%$ greater postimmunization percentage protective titre values.

In the nursing-home residents, there was a statistically significant effect of dose on the antibody response against the $\mathrm{A} / \mathrm{S}$ antigen. After correction for preimmunization titres, there was a 1.3-fold GMT increase when increasing the dose from $10 \mu \mathrm{g}$ to $20 \mu \mathrm{g}$ and a 1.6-fold increase when increasing the dose from $10 \mu \mathrm{g}$ to $60 \mu \mathrm{g}$. The dose effect in postimmunization GMT was not reflected in the postvaccination percentage protective titre parameter, for which the predicted values were 83 for all dose groups. However, statistically significant centre differences between the nursing-home resident populations were found (Table 4).

Surprisingly, in the young age group, the $20 \mu \mathrm{g}$ dose group had a lower response (predicted GMT, 183) than

Table 3 Antibody response to vaccination, according to age and dose

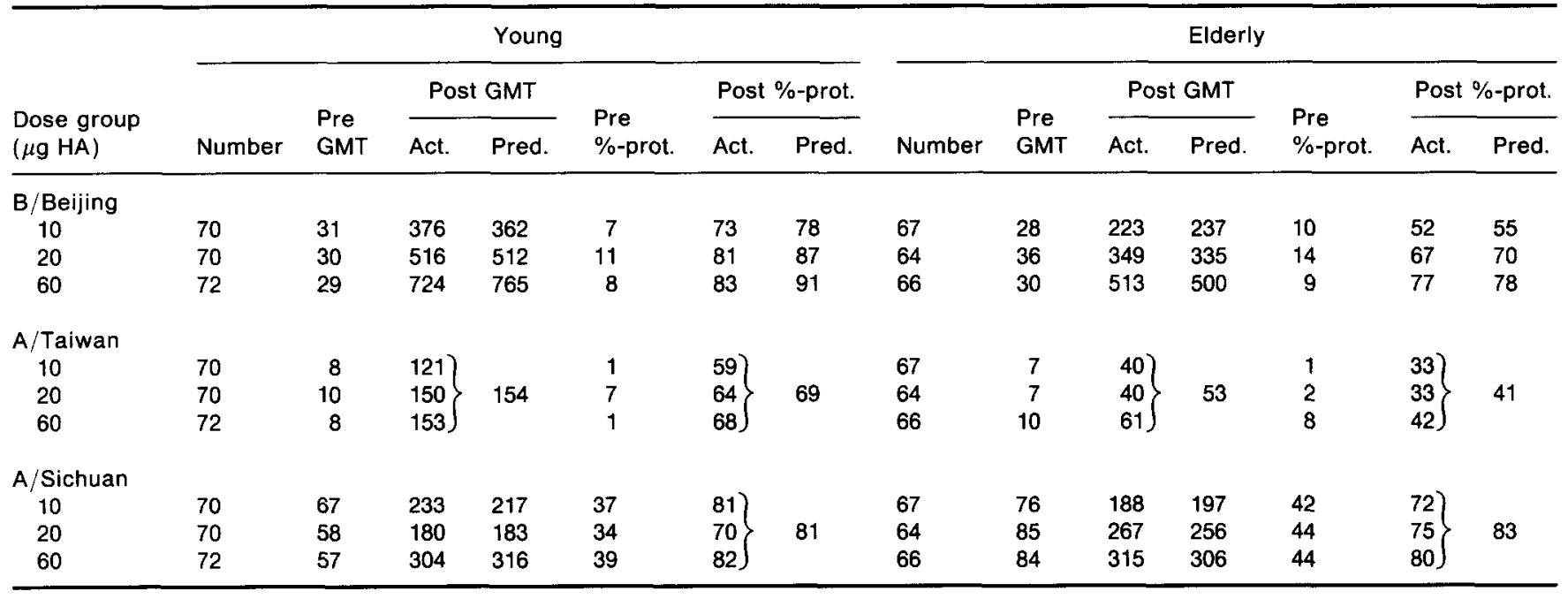

Pre and post refer to prevaccination and postvaccination, respectively. Act., actual; pred., predicted

Prevaccination titre values used in predictions: B/Beijing: 30; A/Taiwan: 10; A/Sichuan (young) 60 , (elderly) 80

Table 4 Differences in antibody response to vaccination between study centres for $A / S i c h u a n$

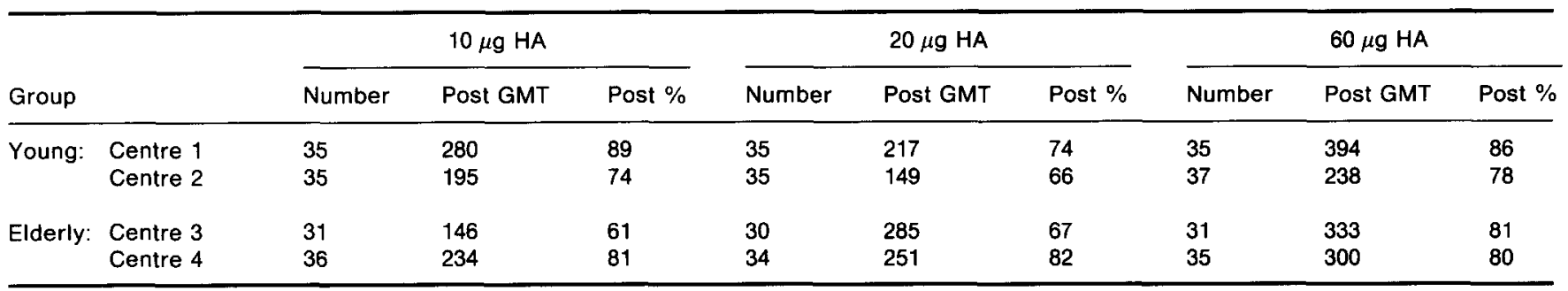

Post GMT: postvaccination GMT; Post \%: postvaccination percentage protection titre 
the $10 \mu \mathrm{g}$ dose group (predicted GMT, 217). As the $60 \mu \mathrm{g}$ dose induced a higher antibody response (predicted GMT, 316) than the $10 \mu \mathrm{g}$, a U-shaped dose-response curve with a 'minimum' at $34-40 \mu \mathrm{g}$ was calculated. This phenomenon could not be explained by differences between centres as it was seen in both study centres (Rotterdam and Paris, Table 4).

\section{Centre differences in the antibody response to vaccination}

In Table 3, the antibody response after active vaccination was shown for the total of both study populations, but not for the four study centres. As indicated, however, there were marked and even highly statistically significant differences between centres 1 and 2 of the young population, as well as between centres 3 and 4 of the nursing-home residents. Despite the observed centre differences for the young for the B/Beijing antigen, the dose-response curves for both centres were similar. Hence, the centre differences did not affect the overall conclusion of a positive dose-response relationship.

In contrast, the dose-response curves in both nursing-home study centres for the A/Sichuan antigen were not similar. Table 4 represents the differences in antibody response between study centres for this antigen. The GMT increase associated with a dose increase from $10 \mu \mathrm{g}$ to $20 \mu \mathrm{g}$ was 2.0 (centre 3 ) and 1.1 (centre 4 ). A dose increase from $20 \mu \mathrm{g}$ to $60 \mu \mathrm{g}$ resulted in a 1.2-fold increase in postimmunization GMT for both study centres. Table 4 also shows that the statistically significant increase in GMT (centre 3) at dose $20 \mu \mathrm{g}$ compared with $10 \mu \mathrm{g}$ is not associated with a significant increase in percentage protective titre $(61$ and $67 \%$, respectively). By increasing the dose to $60 \mu \mathrm{g}$, the percentage protective titre was increased to $81 \%$, a value which was found for all dose groups in the Tel Aviv study centre.

\section{Adverse reactions}

The reported local and systemic adverse reactions following immunization in all dose groups are shown in Table 5. A clear dose-response relationship in the young is demonstrated for the local reactions (predominantly pain on contact) but not for the systemic reactions. In all dose groups, only $11-16 \%$ (placebo) of subjects who reported adverse reactions considered these moderately/ severely inconvenient. The high incidence of reported reactions may be somewhat exaggerated, since medical students are trained to observe very accurately ( $20 \%$ local reactions after placebo).

In striking contrast to the young, in the nursing-home residents no dose-relationship was found for any reactions. Since the assessments were made by the investigators, the low incidence of adverse reactions may

Table 5 Reported adverse effects to immunization, according to age and dose

\begin{tabular}{llllllll}
\hline & \multicolumn{3}{c}{ Young } & & \multicolumn{3}{c}{ Elderly } \\
\cline { 3 - 4 } \cline { 6 - 7 } $\begin{array}{l}\text { Dose } \\
\text { group } \\
(\mu \mathrm{g} \mathrm{HA})\end{array}$ & Number & $\begin{array}{l}\text { Local } \\
(\%)\end{array}$ & $\begin{array}{l}\text { Systemic } \\
(\%)\end{array}$ & & Number & $\begin{array}{l}\text { Local } \\
(\%)\end{array}$ & $\begin{array}{l}\text { Systemic } \\
(\%)\end{array}$ \\
\hline 0 & 70 & 20 & 11 & 65 & 3 & 1 \\
10 & 70 & 44 & 27 & 67 & 1 & 0 \\
20 & 70 & 51 & 17 & 64 & 5 & 2 \\
60 & 72 & 79 & 18 & 66 & 6 & 0 \\
\hline
\end{tabular}

be due to underreporting. Thus, although the figures presented may be either over- or underestimates, the results clearly provide evidence of the tolerance and safety of high doses of the subunit vaccine (total $180 \mu \mathrm{g} \mathrm{HA}$ ) in elderly nursing-home residents.

\section{DISCUSSION}

The current study was performed to investigate whether a relevant increased antibody response in elderly nursing-home residents can be induced by using increased vaccine dosages, so that this important high-risk population can be more effectively protected by immunization against influenza infections ${ }^{7-9}$.

Our data indicate that the issue cannot be addressed for the trivalent vaccine as a whole, but rather should be addressed for each of the vaccine antigens separately. Moreover, the pronounced study centre differences within both populations as found in this large and relatively standardized study, underscore the intrinsic variability of serological influenza vaccination studies, which warrant caution in the interpretation and extrapolation of individual study results. As has been shown previously from a literature review ${ }^{11}$; this variability may often preclude interstudy comparisons of different vaccination studies.

The most consistent dose effect in this study was shown for the B/Beijing antigen (Table 3). By increasing the vaccine dose from 10 to 20 and $60 \mu \mathrm{g} \mathrm{HA}$, the GMT increased approximately 1.4 and 2.1 times, respectively, in both populations.

In contrast to the B/Beijing antigen, we found a lack of a dose effect on the antibody response against the A/Taiwan antigen for both study populations. Increasing the vaccine dose to $60 \mu \mathrm{g} \mathrm{HA}$ did not improve the antibody response. Although twice as many of the young controls reached assumed protective titre levels compared with the nursing-home residents, the flatness of the dose-response curve was very similar for both populations. Hence, the low immune response for the A/Taiwan (H1N1) antigen could not be improved by the use of a higher vaccine dose, as was hypothesized by Gross et al. ${ }^{12}$. The low antibody response for this antigen has also been shown by Iorio et al. ${ }^{24}$ and Gross et al. ${ }^{12}$.

No firm conclusion on the effect of vaccine dose on the antibody response against the $\mathrm{A} /$ Sichuan antigen in nursing-home residents can be drawn from this study, due to statistically significant study centre differences and inconsistent findings. In both study centres of the young volunteers, we found a U-shaped dose-response curve, for which we could not find an explanation. The difference in response following immunizations with 10 and $60 \mu \mathrm{g}$ HA vaccine doses is not considered relevant.

Comparing the antibody response between the two study populations, this study shows a consistent statistically significant difference for the A/Taiwan, but not for the A/Sichuan and B/Beijing antigens. The better response in the young for the $B /$ Beijing antigen was attributed to the higher response values in one of the two young study centres (centre 1). Such strain differences in the effect of age and/or disease on the immune response may also have contributed to the heterogeneous picture which emerged from a literature survey on this subject ${ }^{11}$.

Although various dose-response influenza vaccination trials have been published ${ }^{25-37}$ and have shown relatively flat dose-response curves, only a few of those studies 
have been done in ambulatory elderly and nursing-home residents and these have shown inconsistent data ${ }^{13-15}$. Gross et al. ${ }^{13}$, who studied the immune response of vaccine doses of $15-45 \mu \mathrm{g}$ HA in ambulatory elderly subjects, did not find increasing antibody responses with increasing doses.

Similar findings were reported by Peters et al. ${ }^{14}$, who compared the immune response of 15 and $60 \mu \mathrm{g} \mathrm{HA}$ of the B/USSR/100/83 antigen in non-institutionalized elderly. In contrast, Arden et al. ${ }^{15}$, who also investigated the immune response following immunization with 15 and $60 \mu \mathrm{g}$ HA of the B/USSR/100/83 antigen, reported an improved antibody response in nursing-home residents. The differences between the studies by Peters et al. ${ }^{14}$ and Arden et al. ${ }^{15}$ are of interest, since both studied the same doses of the same antigen. Therefore, it is tempting to speculate that the differences in study results are due to differences in study populations, one being ambulatory elderly and the other nursing-home residents. If this were true, our data are in general agreement with Arden et al. ${ }^{15}$, in that the seroresponse in nursing-home residents can be increased by increasing the dose for the B antigen in the vaccine.

Our data suggest, however, that a major improvement can be achieved by increasing the dose to $20 \mu \mathrm{g} \mathrm{HA}$ and that only marginal further improvements can be achieved by a further dose increase, a finding which cannot be compared with those of Arden et al., since they did not study doses between 15 and $60 \mu \mathrm{g} \mathrm{HA}$. In contrast to Arden $e t$ al., who found a relatively rapid decline of the improved titre levels, we have no information on the duration of the improved immune response for the B/Beijing antigen, since no late serum samples were obtained in our study.

For both influenza A antigens, our data do not yield compelling evidence to justify the expectation of improved vaccine efficacy for nursing-home residents, if the vaccine dose were increased to $60 \mu \mathrm{g} \mathrm{HA}$, although our data were inconsistent for the A/Sichuan antigen.

Although a total dose of $180 \mu \mathrm{g}$ HA subunit vaccine was generally well tolerated by the young and elderly vaccinees in this study, a finding consistent with other reports ${ }^{13-15,38}$ and increased vaccine doses may yield some better immune responses in certain cases, the overall conclusion from our study is that the problem of the relatively low vaccine efficacy in nursing-home residents $^{7-9}$ against influenza infections cannot be adequately solved by increasing the vaccine dose, even up to levels of $60 \mu \mathrm{g} \mathrm{HA}$ /strain. Therefore, the current search for new promising alternative influenza vaccines ${ }^{39,40}$, such as adjuvants ${ }^{41,42}$, liposomes ${ }^{43,44}$, ISCOMS ${ }^{45}$ and CTB-conjugated vaccines ${ }^{46}$ should be continued. Despite the need for more protective influenza vaccines for nursing-home residents, doubts concerning the capacity of the currently available vaccines to reduce influenzaassociated morbidity and mortality are unjustified ${ }^{3-6}$. There is evidence that much is to be gained if the existing vaccines are more widely used in nursing-home residents ${ }^{47}$ as recommended by some public health authorities ${ }^{48}$.

\section{ACKNOWLEDGEMENTS}

The authors are grateful to Dr D. Smith of the University of Kent, Canterbury, for the statistical analysis; Professor R. v. Strik, Erasmus University Rotterdam, for statistical advice; Mrs J. de Ronde, Mrs J. Janssen, Mr R. v. Beek and $\mathrm{Mr}$ G. v.d. Water, for technical assistance, and Mrs C. Vermeulen and Mrs W.J. de Bruijn for preparing the manuscript.

\section{REFERENCES}

1 Recommendations for the prevention and control of influenza during the 1990-91 season. Can. Med. Assoc. J. 1990, 254, 395

2 Editorial. Prevention and control of influenza: Part I, Vaccines. J. Am. Med. Assoc. 1989, 261, 3220

3 Barker, W.H. and Mullooly, J.P. Influenza vaccination of elderly persons: Reduction in pneumonia and influenza hospitalizations and deaths. J. Am. Med. Assoc. 1980, 244, 2547

4 Barker, W.H. and Mullooly, J.P. Effectiveness of inactivated influenza vaccine among non-institutionalized elderly persons. In: Options for the Control of Influenza (Eds Kendal, A.P. and Patriarca, P.A.) Alan R. Liss, 1986, p. 169

5 Patriarca, P.A., Weber, J.A., Parker, R.A., Hall, W.N., Kendal, A.P., Bregman, D.J. and Schonberger, L.B. Efficacy of influenza vaccine in nursing-homes. Reduction in illness and complications during an influenza A/H3N2 epidemic. J. Am. Med. Assoc. 1985, 253, 1136

6 Gross, P.A.,Quinnan, G.V., Rodstein, M., Lamontagne, J.R., Kaslow, R.A., Saah, A.J. et al. Association of influenza immunisation with reduction in mortality in an elderly population: a prospective study. Arch. Int. Med. 1988, 148, 562

7 Arden, N.H., Patriarca, P.A. and Kendal, A.P. Experiences in the use and efficacy of inactivated influenza vaccine in nursing homes. In: Options for the Control of Influenza (Eds Kendal, A.P. and Patriarca P.A.) Alan R. Liss, 1986, p. 155

8 Strassburg, M.A., Greenland, S., Sorvillo, F.J., Lieb, L.E. and Habel, L.A. Influenza in the elderly: report of an outbreak and a review of vaccine effectiveness reports. Vaccine 1986, 4, 38

9 Keren, G., Segev, S., Morag, A., Zakay-Rones, Z., Barzilay, A. and Rubinstein, E. Failure of influenza vaccination in the aged. J. Med. Virol. 1988, 25, 85

10 Ershler, W.B. Influenza vaccination in the elderly: Can efficacy be enhanced? Geriatrics 1988, 43, 79

11 Beyer, W.E.P., Palache, A.M., Baljet, M. and Masurel, N. Antibody induction by influenza vaccines in the elderly: a review of the literature. Vaccine 1989, 7, 385

12 Gross, P.A., Quinnan, G.V., Weksler, M.E., Setia, U. and Douglas, R.G. Relation of chronic disease and immune response to influenza vaccine in the elderly. Vaccine $1989,7,303$

13 Gross, P.A., Quinnan, G.V., Weksler, M.E., Gaerlan, P.F. and Denning, C.R. Immunisation of elderly people with high doses of influenza vaccine. J. Am. Geriatr. Soc. 1988, 36, 209

14 Peters, N.L., Meiklejohn, G. and Jahnigen, D.W. Antibody response of an elderly population to a supplemental dose of influenza B vaccine. J. Am. Geriatr. Soc. 1988, 36, 593

15 Arden, N.H., Patriarca, P.A., Lui, K.J., Harmon, M.W., Brandon, F. and Kendal, A.P. Safety and immunogenicity of a $45 \mu \mathrm{g}$ supplemental dose of inactivated split-virus influenza $B$ vaccine in the elderly. J. Infect. Dis. 1986, 153, 805

16 Berlin, B.S., McQueen, J.L., Minuse, E. and Davenport, F.M. A method for increasing the sensitivity of the haemagglutinationinhibition test with equine influenza virus. Virology 1963, 21, 665

17 Masurel, N., Ophof, B. and de Jong, P. Antibody response to immunisation with influenza A/USSR/77 (H1N1) virus in young individuals primed or unprimed for A/New Jersey/76 (H1N1) virus. J. Hyg. 1981, 87, 201

18 Masurel, N. and Laufer, J. A one year study of trivalent influenza vaccines in primed and unprimed volunteers: immunogenicity, clinical reactions and protection. J. Hyg. 1984, 92, 263

19 Beyer, W.E.P., Teunissen, M.W.E., Diepersloot, R.J.A. and Masurel, N. Immunogenicity and reactogenicity of two doses of a trivalent influenza split vaccine. An open randomized study in healthy, unprotected, adult volunteers. J. Drug Ther. Res. 1986, 11, 369

20 SAS/STAT User's Guide Volume 2, Version 6, Fourth Edition, SAS Institute Inc., Cary, North Carolina

21 Voth, D.W., Feldman, H.A. and Steinschneider, A. Comparative responses of elderly persons to aqueous and depot influenza vaccines. Arch. Environ. Health 1966, 13, 576

22 Ada, G.L. and Jones, P.D. The immune response to influenza infection Curr Topics Microbiol Immunol. 1986, 128, 1

23 Couch, R.B. and Kasel, J.A. Immunity to influenza in man. Annu. Rev. Microbiol. 1983, 37, 529

24 Iorio, A.M., Rivosecchi, P., Zei, T., Neri, M. and Merlett, L. Immune 
response to trivalent inactivated influenza vaccine in young and elderly subjects. Vaccine $1989,7,341$

25 Mostow, S.R., Schoenbaum, S.C., Dowdle, W.R., Coleman, M.T. and Kaye, H.S. Inactivated vaccines. I. Volunteer studies with very high doses of influenza vaccine purified by zonal ultracentrifugation. Postgrad. Med. J. 1973, 49, 152

26 Feery, B.J., Hampson, A.W., Fortsyth, J.R.L. and Evered, M.G. Effect of dose on antibody response to subunit influenza vaccine. Med. $J$. Austr. 1977, 2, 324

27 Potter, C.W., Jennings, R., Phair, J.P., Clarke, A. and Stuart-Harris C.H. Dose-response relationship after immunisation of volunteers with a new surface-antigen-adsorbed influenza virus vaccine. J. Infect. Dis. 1977, 135, 423

28 Goodeve, A., Potter, C.W., Clark, A., Jennings, R., Schild, G.C. and Yetts, R.A. Graded-dose study of inactivated surface antigen influenza $B$ vaccine in volunteers: reactogenicity, antibody response and protection to challenge virus infection. J. Hyg. (Camb.) 1983, so, 107

29 Quinnan, G.V., Schooley, R. Dolin, R., Ennis, F.A. and Gwaltney, J.M Serologic responses and systemic reactions in adults after vaccination with monovalent $A /$ USSR/77 and trivalent $A / U S S R / 77$, B/Hong Kong/72 influenza vaccines. Rev. Infect. Dis. 1983, 5, 748

30 Jennings, R., Smith, T.L., Mellersh, A.R., Clark, A., Spencer, R.C. and Potter, C.W. Antibody response and persistence in volunteers following immunisation with varying dosages of a trivalent surface antigen influenza virus vaccine. J. Hyg. (Camb.) 1985, 94, 87

31 La Montagne, J.R., Noble, G.R., Quinnan, G.V., Curlin, G.T. Blackwelder, W.C., Smith, J.I. et al. Summary of clinical trials of inactivated influenza vaccine-1978. Rev. Infect. Dis. 1983, 5, 723

32 Parkman, P.D., Galasso, G.J., Top, F.H. and Noble, G.R. Summan of clinical trials of influenza vaccines. J. Infect. Dis. 1976, 134, 100

33 Nicholson, K.G., Tyrrell, D.A.J., Harrison, P., Potter, C.W. Jennings, R., Clark, A. et al. Clinical studies of monovalent inactivated whole virus and subunit A/USSR/77 (H1N1) vaccine: serological responses and clinical reactions. J. Biol. Stand. 1979 , 7, 123

34 Cate, T.R., Kasel, J.A., Cough, R.B., Six, H.R. and Knight, V. Clinical trials of bivalent influenza $A / N e w$ Jersey/76-A/Victoria/75 vaccines in the elderly. J. Infect. Dis. 1977, 136S, S518

35 Cate, T.R., Couch, R.B., Kasel, J.A. and Six, H.R. Clinical trials of monovalent influenza $\mathrm{A} / \mathrm{New}$ Jersey/76 virus vaccines in adults: Reactogenicity, antibody response and antibody persistence. J. Infect. Dis. 1977, 1365, S450

36 Gross, P.A., Quinnan, G.V., Gaerlan, P.F., Denning, C.R., Davis, A. Lazicki, M. and Bernius, M. Potential for single high-dose influenza immunisation in unprimed children. Pediatrics 1982, 70, 982

37 Sullivan, K.M., Monto, A.S. and Foster, D.A. Antibody response to inactivated influenza vaccines of various antigenic concentrations. J. Infect. Dis. 1990, 161, 333

38 Margolis, K.L., Nichol, K.L., Poland, G.A. and Pluhar, R.E. Frequency of adverse reactions to influenza vaccine in the elderly. J. Am. Med. Assoc. 1990, 264, 1139

39 Reichelderfer, P.S. and Kendal, A.P. Influenza control: New vaccines and antivirals with broad efficacy against influenza virus are needed. Drug News and Perspectives 1989, 2, 99

40 Melnick, J.L. Virus vaccines: principles and prospects. Bull. WHO 1989, 7, 257

41 Palache, A.M., Masihi, K.N. and Masek, K. Effect of Adamantylamide Dipeptide on antibody response to influenza subunit vaccines and protection against aerosol influenza infection. In: Immunotherapeutic Prospects of Infectious Diseases (Eds Masihi, K.N. and Lange, W.) Springer Verlag, Berlin, Heidelberg, 1990, p. 347

42 Allison, A.C. and Byars, N.E. An adjuvant formulation that selectively elicits the formation of antibodies of protective isotypes and of cell-mediated immunity. J. Immunol. Meth. 1986, 95, 157

43 Guink, N.E., Kris, R.M., Goodman-Snitkoff, G., Small, P.A. and Mannino, R.J. Intranasal immunisation with proteoliposomes protects against influenza virus vaccine in mice. Vaccine 1989, 7, 147

44 Gregoriadis, G. Liposomes for drugs and vaccines. Trends Biotechnol. 1985, 3, 235

45 Sundquist, B., Lovgren, K. and Morein, B. Influenza virus ISCOMS: antibody response in animals. Vaccine 1988, 6, 49

46 Tamura, S. and Samegai, Y. Enhancement of protective antibody responses by cholera toxin $B$ subunit inoculated intranasally with influenza vaccine. Vaccine 1989, 7, 257

47 Anderson, R.M. and May, R.M. Vaccination and herd immunity to infectious diseases. Nature 1985, 318, 323

48 Recommendations of the Immunisation Practices Advisory Committee (ACIP). Prevention and control of influenza. Morb. Mort. Week. Rep. 1987, 36, 373 